\title{
The Effects of Apelin on Mesenteric Ischemia and Reperfusion Damage in an Experimental Rat Model
}

\author{
Tamer Sağıroğlu', Serhat Oğuz', Gönül Sağıroğlu², Elif Çopuroğlu², Tulin Yalta³, Mustafa Burak Sayhan, Mehmet Ali Yağcı ${ }^{5}$ \\ ${ }^{1}$ Department of General Surgery, Faculty of Medicine, Trakya University, Edirne, Turkey \\ ${ }^{2}$ Department of Anesthesiology and Reanimation, Faculty of Medicine, Trakya University, Edirne, Turkey \\ ${ }^{3}$ Department of Pathology, Faculty of Medicine, Trakya University, Edirne, Turkey \\ ${ }^{4}$ Department of Emergency, Faculty of Medicine, Trakya University, Edirne, Turkey \\ ${ }^{5}$ Department of General Surgery, Hakkari Goverment Hospital, Hakkari, Turkey
}

\section{ABSTRACT}

Objective: Intestinal ischemia-reperfusion (I/R) injury is associated with high morbidity and mortality rates. There is ongoing research to find an effective preventive or treatment agent. We aimed to evaluate the effects of apelin 13 (AP) on intestinal I/R injury in a rat model.

Material and Methods: Twenty-four male Sprague-Dawley rats aged 6-8 weeks and weighing $280 \pm 20 \mathrm{~g}$ were equally divided into three groups (control, $\mathrm{I} / \mathrm{R}$ and $\mathrm{I} / \mathrm{R}+\mathrm{AP}$ ). The control group underwent superior mesenteric artery (SMA) mobilization alone without any clamping. In the $\mathrm{I} / \mathrm{R}$ and $\mathrm{I} / \mathrm{R}+\mathrm{AP}$ groups, an atraumatic microvascular bulldog clamp was placed across the SMA at its point of origin from the aorta. In the I/R+AP group, $2 \mu \mathrm{g} / \mathrm{kg} / \mathrm{d}$ apelin was administered intraperitoneally. After 60 minutes of ischemia, relaparotomy was performed to remove the microvascular clamp on the SMA for 3 hours of reperfusion. After 3 hours, tissue samples were obtained for biochemical [malondialdehyde (MDA) and glutathione (GSH) levels] and histopathological analyses.

Results: MDA levels were significantly higher in the I/R group compared to the control group. Although MDA levels were lower in the I/R+AP group compared tothe I/R group, the difference was not statistically significant. There was also no significant difference between the I/R+AP and I/R groups regarding GSH levels. The median histopathological grade was significantly lower in the I/R+AP group compared to the $\mathrm{I} / \mathrm{R}$ group ( $p=0.001$ ).

Conclusion: Apelin appeared to have a positive effect on oxidative injury; this did not reach statistical significance. Thus, the role of apelin and associated findings in the initial treatment of intestinal ischemia needs further large-scale animal studies before human use.

Key Words: Apelin, mesenteric ischemia, reperfusion injury

\section{Introduction}

Tissue ischemia is a key event in clinical conditions such as myocardial infarction and stroke and may also occur as a complication following surgical procedures. Tissue damage due to ischemia is paradoxically further increased after reperfusion. This negative effect of reperfusion especially becomes more pronounced in cases of prolonged ischemia and is caused by a series of inflammatory reactions, also known as ischemia-reperfusion (I/R) injury (1). The pathophysiology of I/R injury is extremely complex including vascular endothelial cells, leucocytes, oxygen radicals, inflammatory mediators such as platelet-activating factor and tumor necrosis factor, and adhesion molecules (2).

The small intestine is probably the most sensitive visceral organ to I/R injury. Intestinal I/R injury is associated with high morbidity and mortality rates in trauma and surgical patients (3). Conditions causing any kind of interruption and/ or reduction of intestinal blood flow lead to I/R injury. These conditions may include abdominal aortic aneurysm surgery, cardiopulmonary bypass, strangulated hernias, neonatal nec- rotizing enterocolitis, intestinal transplantation, and septic and hypovolemic shock. Interruption of blood supply rapidly results in ischemic injury in the metabolically active intestinal tissue and, as well as restoration of blood flow, paradoxically leads to reperfusion injury (3), which is even greater than the initial damage caused by ischemia itself, as a result of a series of events. As intestinal mucosa is a critical site where several acute-phase proteins, hormones, and cytokines are synthesized, intestinal damage is not limited to this region and results in impairment of functions and integrity of distant organs as well. Moreover, bacterial translocation due to intestinal damage may lead to further deterioration of the clinical picture by causing sepsis, shock, and multiple organ failure (3).

Considerable increase in surgical interventions and organ transplantations in recent years and high morbidity and mortality rates associated with ischemia-related disorders have led to an increase in interest regarding I/R injury. Currently, no measures have been shown to be effective in the prevention or treatment of I/R injury, and there are ongoing studies investigating different potential agents. In the present study, the effects of apelin 13 (AP) on intestinal I/R injury were investigated in a rat model. 


\section{Material and Methods}

\section{Study design}

This study was approved by the Animal Ethics Committee and conducted at the Laboratory Animals Care Unit in accordance with the guidelines for the care and use of laboratory animals. Twenty four male Sprague-Dawley rats aged 6 to 8 weeks and weighing $280 \pm 20 \mathrm{~g}$ were used. Using a computer generated table of random numbers, rats were randomly assigned to the following groups: control group (group $C, n=8$ ), ischemia-reperfusion group (group $I / R, n=8$ ), and ischemiareperfusion+apelin group (group $I / R+A P, n=8$ ). The animal room was maintained at a temperature of $22 \pm 2^{\circ} \mathrm{C}$ and a relative humidity of $55 \pm 15 \%$, with a 12 -hour light-dark cycle. Tap water and chow were freely available throughout the acclimatization and study periods.

\section{Chemicals and Reagents}

Apelin-13 (Apelin ${ }^{\circledR}$, Phoenix Pharmaceutical, Belmont, CA, USA) was commercially purchased. Apelin was administered intraperitoneally beginning three days prior to the surgical procedure in group I/R+AP at a dose of $2 \mu \mathrm{g} / \mathrm{kg} /$ day as previously described by Petrescu (4). The same amount of normal saline was administered intraperitoneally in groups $\mathrm{I} / \mathrm{R}$ and $\mathrm{C}$.

\section{Surgical Procedure}

After an overnight fasting period, each animal was anesthetized by intramuscular administration of $5 \mathrm{mg} / \mathrm{kg}$ of xylazine (Rompun, Bayer Ilac Sanayi, Sisli, Istanbul, Turkey) and 30 $\mathrm{mg} / \mathrm{kg}$ of ketamine hydrochloride (Ketalar, Eczacibasi llac San, Istanbul, Turkey). Abdomen of each rat was then shaved and cleansed by povidone iodine solution (Isosol, Merkez Laboratory, Ilac San, Istanbul, Turkey). Using a sterile technique, all rats underwent laparotomy through a $3 \mathrm{~cm}$ midline incision. The aorta and visceral arteries were exposed in the abdominal cavity, and the ligament of Treitz was also incised to better expose the superior mesenteric artery (SMA). Group C underwent SMA mobilization only without any clamping. In groups I/R and I/R+AP, an atraumatic microvascular bulldog clamp was placed across the SMA at its point of origin from the aorta, with special care to avoid occlusion of the superior mesenteric vein. Mesenteric ischemia was confirmed by noting loss of mesenteric pulsations and observing intestinal paleness. The bowel was then returned to the abdominal cavity, and the area of incision was closed by interrupted atraumatic 4.0 silk sutures. After 60 minutes of ischemia, relaparotomy was performed to remove the microvascular clamp on the SMA for 3 hours of reperfusion. Mesenteric reperfusion was confirmed by noting the restoration of pulsations and intestinal color. The bowel was again returned to the abdominal cavity, and the area of incision was closed with 4.0 silk sutures. The bowel was left in the abdomen during reperfusion. At the end of 3 hours, tissue samples were obtained from an area of small intestine $5 \mathrm{~cm}$ proximal to the ileocecal region. The bowel specimens from each animal were harvested for both biochemical and histopathological analysis. The tissues were rinsed with cold saline solution and all of the tissue specimens were fixed in $10 \%$ buffered formalin for histopathological analysis.

\section{Malondialdehyde and Glutathione assays}

The bowel tissue samples were washed with physiological saline and kept in a freezer until the day of the experiment. These samples were homogenized with $150 \mathrm{mmol} / \mathrm{L}$ ice-cold $\mathrm{KCl}$ for the measurement of malondialdehyde (MDA) and glutathione (GSH) levels. Homogenates were centrifuged at $2600 \times \mathrm{g}$ for $10 \mathrm{~min}$ at $4^{\circ} \mathrm{C}$. The MDA concentrations in the renal tissue, which is an indicator of lipid peroxidation, were assayed in the form of thiobarbituric acid-reacting substances (5). Then, $0.2 \mathrm{~mL}$ of $8.1 \%$ sodium dodecyl sulphate, $1.5 \mathrm{~mL}$ of $20 \%$ acetic acid, $1.5 \mathrm{~mL}$ of $0.8 \%$ thiobarbituric acid, and $0.6 \mathrm{~mL}$ of distilled water were added to the supernatant. This mixture was heated to $95^{\circ} \mathrm{C}$ for $60 \mathrm{~min}$. After cooling with tap water, $1.0 \mathrm{~mL}$ of distilled water and $5.0 \mathrm{~mL}$ of a mixture of $\mathrm{n}$-butanol: pyridine $(15: 1, \mathrm{v} / \mathrm{v})$ were added and the mixture was shaken vigorously and centrifuged at $2600 \times \mathrm{g}$ for $10 \mathrm{~min}$ at $25^{\circ} \mathrm{C}$. The absorbance of the organic layer was read at 532 $\mathrm{nm}$. Malondialdehyde was quantified using an extinction coefficient of $1.56 \times 105 \mathrm{~L} / \mathrm{mol}$ per $\mathrm{cm}$ and expressed as nmol MDA/ $\mathrm{mg}$ tissue. The glutathione level was determined by the Ellman method (6). The concentration of GSH was monitored spectrophotometrically at $412 \mathrm{~nm}$, and the results were expressed as $\mu \mathrm{mol} / \mathrm{g}$ tissue.

\section{Histopathological Examination}

Tissue specimens were fixed in $10 \%$ formalin for 24 hours, then embedded in paraffin and sliced into $5 \mu \mathrm{m}$ sections. Slides were stained with hematoxylin and eosin (H\&E) and examined under a light microscope. Each slide was evaluated by two expert investigators blinded to the experiment and data. Intestinal injury was classified into a five-tiered scale defined by Chiu et al. (7) as grade 0: no diagnostic change; grade 1: subepithelial layer lifting from the lamina propria, usually at the apex of the villus; grade 2: moderate epithelial cell layer lifting from the lamina propria; grade 3: loss of a few villi with massive epithelial lifting from the lamina propria with a few denuded villi; and grade 4: disintegration of the lamina propria with ulceration and hemorrhage.

\section{Statistical analysis}

Data were analyzed using SPSS 15.0 (SPSS Inc., Chicago, IL, USA) for Windows. Histopathological findings of the groups were compared using the Kruskal-Wallis test. For group comparisons, the Mann-Whitney $U$ test was used. Bonferroni correction was used to adjust the $p$ value due to multiple comparisons, and a $p$ value less than 0.017 was considered significant.

\section{Results}

Serum MDA and GSH levels of the study groups are presented in Table 1. There was a significant difference between the groups in terms of MDA levels. Serum MDA level in group $C$ was significantly lower than those in group I/R and group $1 / R+A P(p=0.001$ and $p=0.008$, respectively). However, there was no significant difference between group I/R and group I/ $R+A P$ regarding MDA level $(p=0.294 ;$ Figure 1$)$. Moreover, no significant difference was found between the study groups in terms of GSH level (Figure 2). 
No pathological changes were noted on histopathological evaluation in any of the subjects in the control group. Grade 4 intestinal damage was observed in all of the subjects in I/R group. Whereas grade 0 damage was noted in 3 , grade 2 damage was noted in 2, grade 3 damage was noted in 2, and grade 4 damage was noted in one subject among 8 subjects in group I/R+AP (Figure 3). According to these histopathological results, median damage grade was 4 in the I/R group and 2 in the $I / R+A P$ group and the difference was significant $(p=0.001)$ (Figure 4).

\section{Discussion}

Mesenteric ischemia constitutes about $0.1 \%$ of all hospital admissions and $1-2 \%$ of admissions due to abdominal pain. Despite the increase of knowledge on mesenteric ischemia, it is still associated with a high mortality rate (8). Being a branch of the abdominal aorta, SMA supplies a large part of the intestine, from the duodenum to distal transverse colon. Pathological conditions causing an interruption or reduction of blood flow in SMA may lead to mesenteric ischemia. The major goal of the treatment is restoration of blood flow as soon as possible. During the period of interrupted blood flow, ischemic damage occurs in two stages. The first stage begins immediately after ischemia and continues for a period of 2-3 hours. The second stage begins 12-24 hours after ischemia and lasts about 3-4 days. Clinical presentation may vary depending on the duration of ischemia. Cytokines and acute phase proteins released from the intestinal mucosa during reperfusion following ischemia causes dissemination of the pathological process beyond the intestinal mucosa and affects distant organs. Subsequently, cell death and organ failure occurs. Therefore, there has recently been a growing amount of interest in this severe clinical condition resulting from I/R injury (8).

Several agents have been investigated for the prevention and/or treatment of mesenteric I/R injury. Most of the information has been established from experimental studies using animal models. Agents under investigation include antioxidants, several amino acids, phospholipids, hormones, polyphones and flavonoids, herbal extracts, pharmacological agents, carbon monoxide inhalation, erythropoietin, statins, and hyperbaric oxygen $(8,9)$.

It has been demonstrated in an experimental study that intravenous immunoglobulin has a protective effect against mesenteric I/R damage in rats (10). In another study using a rat model, it has been reported that hypocapnia reduces I/R injury despite having direct harmful effects on the liver (11). It has also been shown that alpha-melanocyte-stimulating hormone (MSH) significantly limits postischemic injury in rat small intestine (12). Furthermore, pyruvate infusion has been reported to have a local protective effect against I/R injury in

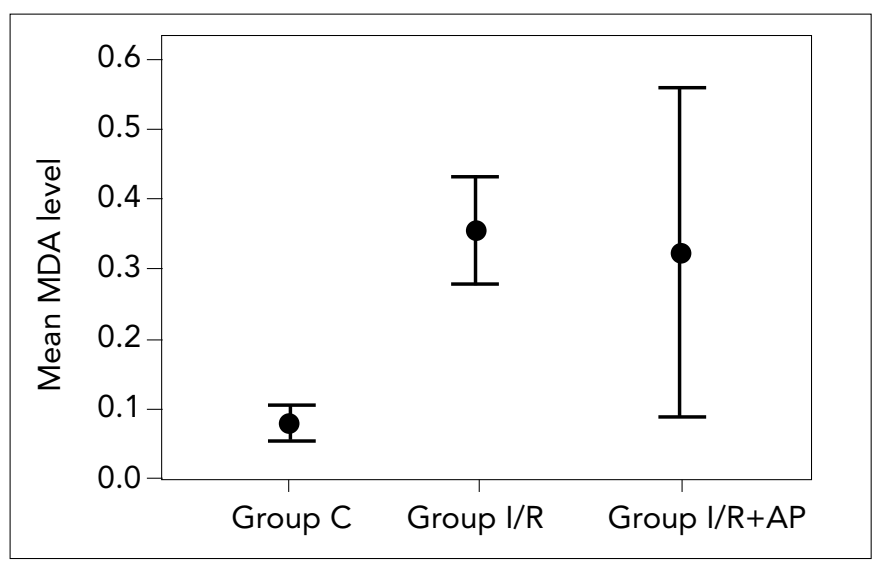

Figure 1. The MDA levels of study groups

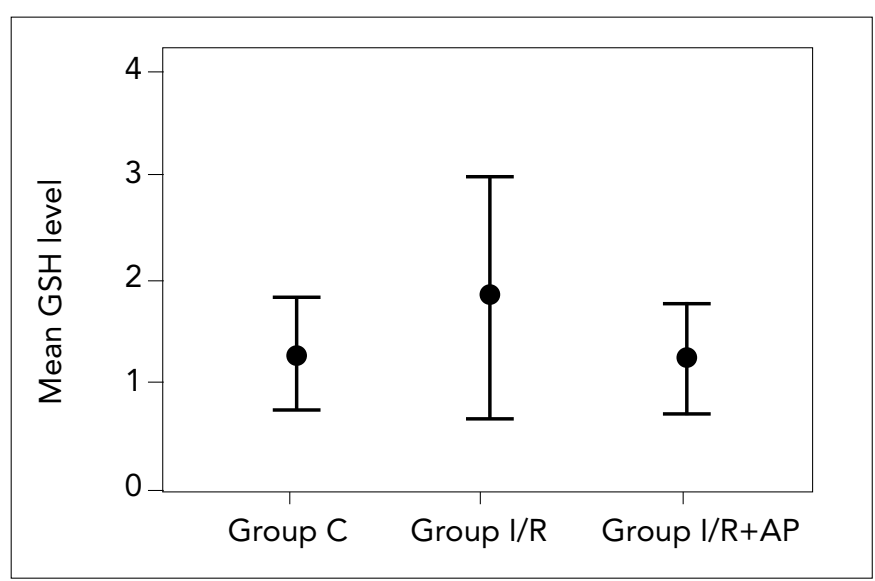

Figure 2. The GSA levels of study groups

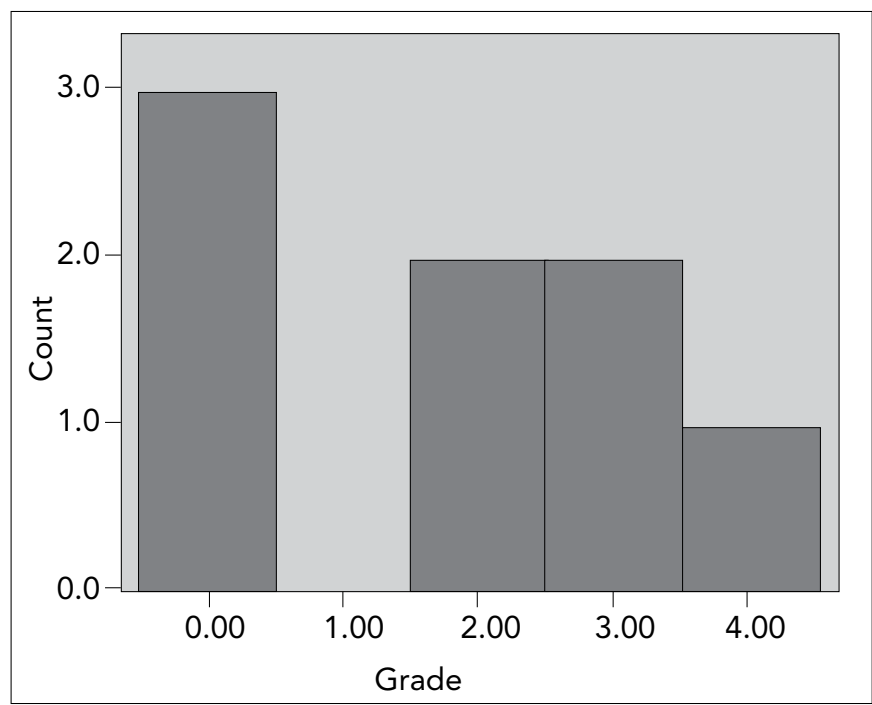

Figure 3. The distribution of the intestinal injury in Group I/R+AP

Table 1. The MDA and GSA levels of study groups

\begin{tabular}{|c|c|c|c|c|}
\hline & Group C & Group I/R & Group I/R+AP & $p$ \\
\hline MDA (nmol/g fresh tissue) & $0.08 \pm 0.03$ & $0.36 \pm 0.09$ & $0.32 \pm 0.28$ & 0.002 \\
\hline GSH ( $\mu \mathrm{mol} / \mathrm{g}$ fresh issue) & $1.27 \pm 0.60$ & $1.83 \pm 1.42$ & $1.23 \pm 0.62$ & 0.755 \\
\hline
\end{tabular}




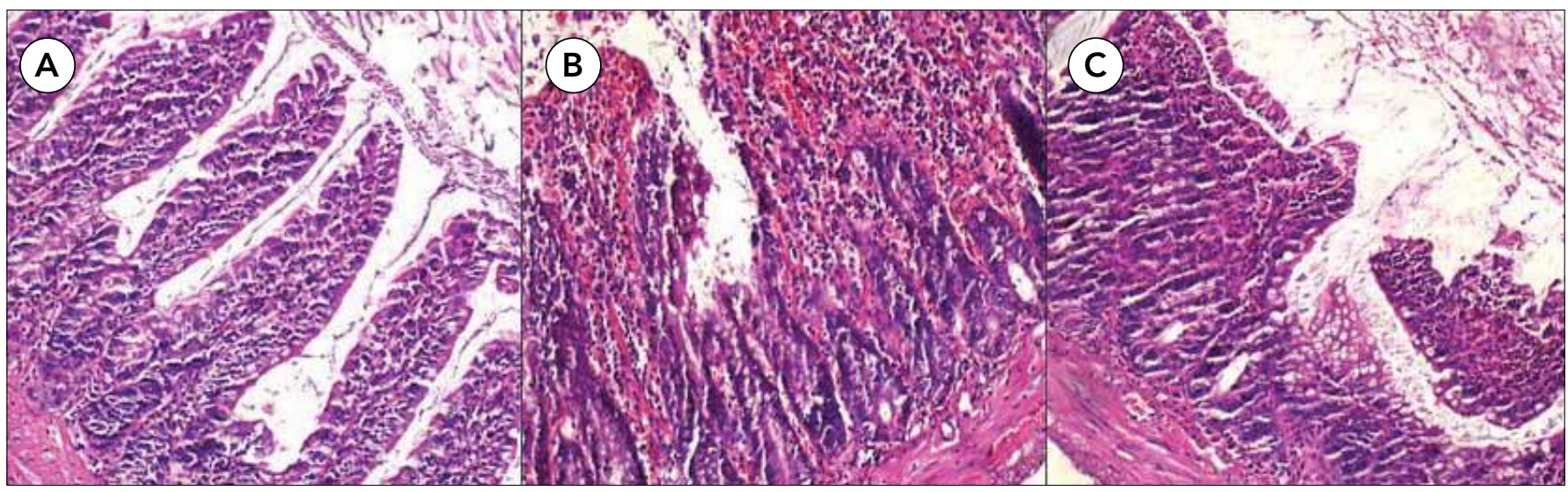

Figure 4. A. The histopathologic section of control group demonstrating normal architecture (HEX100). B. The histopathologic section showing prominent ulceration and hemorrhage in the ischemia group (HEx100). C. Slight epithelial layer lifting at the apex of the villus in apelin treated group (HEX100)

rat small intestine (13). It has been shown in a rat model that proanthocyanidin has a protective effect against mesenteric I/R injury both at intestinal and distant organ level (14). It has also been reported that application of pyrolidine dithiocarbamate prevents mesenteric I/R injury in rats (15).

Apelin has been shown to play a significant role in the regulation of cardiovascular functions and fluid homeostasis. Apelin receptor (APJ) mRNA can be detected in several human organs including the brain, spleen, thymus, prostate, testis, ovary, small and large intestines (16). It has been demonstrated that exogenous apelin treatment has a protective effect against myocardial I/R injury in animal models (17-19). In the study by Zeng et al. (19), in which the Langendorff model of myocardial I/R injury was used in adult male rats, $20 \mathrm{~min}$ stabilization followed by 40 min global ischemia (achieved by total perfusion arrest) and then 30 min reperfusion were performed on isolated hearts. They reported a cardioprotective effect in the group in which they used a buffer containing AP. They also demonstrated the protective effect of apelin in a cell culture model using primary neonatal rat cardiomyocytes. We also investigated the effects of apelin on mesenteric I/R injury in a rat model. We found that the median histopathological grade was significantly lower in the $I / R+A P$ group compared to the I/R group.

It is known that oxidative stress induced by ischemia leads to formation of GSH and MDA $(20,21)$. Turnage et al. (21) evaluated hepatic tissue following experimentally induced intestinal I/R injury for lipid peroxidation products, and oxidized and reduced glutathione. They demonstrated that oxidized glutathione was increased significantly following 30 and 60 min of reperfusion; however, they reported no increase in any of the products of lipid peroxidation. In the present study, we also measured GSH and MDA levels to evaluate ischemia induced oxidative damage in tissues, but failed to demonstrate this damage through these levels.

\section{Conclusion}

Although we demonstrated positive effects of apelin on experimentally induced I/R injury at histopathological level, we did not found any positive effects on oxidative injury. Even though apelin appeared to have a positive effect on oxidative injury, this did not reach statistical significance. It should be noted, however, that the role of this adipocytokine in the initial treatment of intestinal ischemia needs further investigation, and associated findings need to be confirmed by future large scale animal model studies before being tested in clinical conditions in humans. Furthermore, the clinical picture resulting from I/R injury is still associated with high morbidity and mortality rates. Studies investigating the effectiveness of novel pharmacological agents are needed in order to reduce morbidity and mortality.

\section{Conflict of Interest}

No conflict of interest was declared by the authors.

\section{References}

1. Diepenhorst GM, van Gulik TM, Hack CE. Complement-mediated ischemia-reperfusion injury: lessons learned from animal and clinical studies. Ann Surg 2009;249:889-99. [CrossRef]

2. Carden DL, Granger DN. Pathophysiology of ischaemia-reperfusion injury. J Pathol 2000;190:255-66. [CrossRef]

3. Mallick IH, Yang W, Winslet MC, Seifalian AM. Ischemia-reperfusion injury of the intestine and protective strategies against injury. Dig Dis Sci 2004;49:1359-77. [CrossRef]

4. Petrescu BC, Gurzu B, lancu RI, Indrei A, Dumitriu I, Chelaru L, et al. Apelin effects on lipopolysaccharide-increased pulmonary permeability in rats. Rev Med Chir Soc Med Nat lasi 2010;114:163-9.

5. Ohkawa H, Ohishi N, Yagi K. Assay for lipid peroxides in animal tissues by thiobarbutiric acid reaction. Anal Biochem 1979;95:351-8. [CrossRef]

6. Ellman GL. Tissue sulfhydryl groups. Arch Biochem Biophys 1959;82:70-7. [CrossRef]

7. Chiu CJ, McArdle AH, Brown R, Scott HJ, Gurd FN. Intestinal mucosal lesion in low-flow states. I. A morphological, hemodynamic, and metabolic reappraisal. Arch Surg 1970;101:478-83. [CrossRef]

8. Martinez JP, Hogan GJ. Mesenteric ischemia. Emerg Med Clin North Am 2004;22:909-28. [CrossRef]

9. Chatterjee PK. Novel pharmacological approaches to the treatment of renal ischemia-reperfusion injury: a comprehensive review. Naunyn Schmiedebergs Arch Pharmacol 2007;376:1-43. [CrossRef]

10. Anderson J, Fleming SD, Rehrig S, Tsokos GC, Basta M, SheaDonohue T. Intravenous immunoglobulin attenuates mesenteric 
ischemia-reperfusion injury. Clin Immunol 2005;114:137-46. [CrossRef]

11. Duggan M, Engelberts D, Jankov RP, Worrall JM, Qu R, Hare GM, et al. Hypocapnia attenuates mesenteric ischemia-reperfusion injury in a rat model. Can J Anaesth 2005;52:262-8. [CrossRef]

12. Hassoun HT, Zou L, Moore FA, Kozar RA, Weisbrodt NW, Kone BC. Alpha-melanocyte-stimulating hormone protects against mesenteric ischemia-reperfusion injury. Am J Physiol Gastrointest Liver Physiol 2002;282:G1059-68.

13. Petrat $F$, Rönn $T$, de Groot $H$. Protection by pyruvate infusion in a rat model of severe intestinal ischemia-reperfusion injury. J Surg Res 2011;167:e93-e101. [CrossRef]

14. Sizlan A, Guven A, Uysal B, Yanarates O, Atim A, Oztas E, Cosar A, Korkmaz A. Proanthocyanidin protects intestine and remote organs against mesenteric ischemia/reperfusion injury. World $\mathrm{J}$ Surg 2009;33:1384-91. [CrossRef]

15. Teke Z, Kabay B, Aytekin FO, Yenisey C, Demirkan NC, Sacar $M$, et al. Pyrrolidine dithiocarbamate prevents 60 minutes of warm mesenteric ischemia/reperfusion injury in rats. Am J Surg 2007;194:255-62. [CrossRef]
16. Kleinz MJ, Davenport AP. Emerging roles of apelin in biology and medicine. Pharmacol Ther 2005;107:198-211. [CrossRef]

17. Kleinz MJ, Baxter GF. Apelin reduces myocardial reperfusion injury independently of PI3K/Akt and P70S6 kinase. Regul Pept 2008;146:271-7. [CrossRef]

18. Simpkin JC, Yellon DM, Davidson SM, Lim SY, Wynne AM, Smith CC. Apelin-13 and apelin-36 exhibit direct cardioprotective activity against ischemia-reperfusion injury. Basic Res Cardiol 2007;102:518-28. [CrossRef]

19. Zeng XJ, Zhang LK, Wang HX, Lu LQ, Ma LQ, Tang CS. Apelin protects heart against ischemia/reperfusion injury in rat. Peptides 2009;30:1144-52. [CrossRef]

20. Janssen M, Koster JF, Bos E, de Jong JW. Malondialdehyde and glutathione production in isolated perfused human and rat hearts. Circ Res 1993;73:681-8. [CrossRef]

21. Turnage RH, Bagnasco J, Berger J, Guice KS, Oldham KT, Hinshaw DB. Hepatocellular oxidant stress following intestinal ischemia-reperfusion injury. J Surg Res 1991;51:467-71. [CrossRef] 